\title{
Strain and Strain Rate To Evaluate Right Heart Function of Ebstein Anomaly (EA) Patients Before and After Operation
}

\author{
Xiang-Jun Liu, Lian-Yi Wang, Hong-Yin Li, Jian Cui, Rui Liu \\ Heart Center, The First Hospital of Tsinghua University (Beijing Huaxin Hospital), Beijing, China
}

\section{ABSTRACT}

Objective: This study was aimed to elucidate the feasibility of using right ventricular (RV) strain and strain rate to evaluate right heart function of Ebstein anomaly (EA) patients before and after operation.

Methods: Sixty EA patients and 30 healthy controls underwent echocardiography (UCG) for evaluation of right heart function. Preoperative UCG and 1-week and 3-month postoperative UCG were performed in EA patients. RV strain and strain rate were measured on the four-chamber section of tissue Doppler imaging (TDI).

Results: The strain and strain rate representative of right ventricle systolic function were reduced prior to operation. RV strain and strain rate improved after the operation $(P<.001)$, most significantly in the basal segment and middle segment of the free wall of the right ventricle as well as the basal segment of the interventricular septum $(P<.001)$.

Conclusions: The measurement of RV strain and strain rate on tissue Doppler imaging can be employed to assess the preoperative and postoperative RV function, proves the positive effect of tricuspid valve repair on right heart function, and offers more insight on right heart function evaluation.

\section{INTRODUCTION}

Ebstein anomaly is known to have significant anatomical variation while surgery is highly dependent on anatomy. Due to the intricacy of operation, there is no consensus yet on the surgical approach to EA correction. The treatment plan rests on the severity of tricuspid valve impairment. Two-hundredthirty-seven patients [Zhang 2018] with Ebstein anomaly received surgical procedures in our hospital. The principle of the techniques is to anatomically reconstruct the tricuspid valve and right ventricle. For most cases, the anatomical repair was demonstrated with low mortality, less complications, and excellent durability at long-term follow up. Late survival was $99.1 \%$. Indication of tricuspid valve regurgitation improved from $3.6 \pm 0.3$ to $1.5 \pm 0.4$. Survival rate at 5 and 10 years was

Received April 25, 2020; accepted fuly 14, 2020.

Correspondence: Lian-Yi Wang, Heart Center, The First Hospital of Tsinghua University (Beijing Huaxin Hospital), No. 6 Fiuxianqiao Yijiefang, Chaoyang District Beijing, 100016, China; +86-010-64308218 (e-mail: lywang@mail. tsingbua.edu.cn).
$98.6 \%$ and $98.2 \%$, respectively. Other relevant conditions, such as right ventricular enlargement and right ventricular atrialization, predispose to thrombosis and more deleterious hemodynamic status. Therefore, it is important to study right ventricular function after the operation and stratify the disease severity prior to EA correction. Thus, this study aims to assess the feasibility of using RV strain and strain rate to evaluate right heart function of EA patients before and after operation.

\section{MATERIALS AND METHODS}

Subjects (EA group): Sixty patients, who underwent Ebstein anomaly correction in our hospital, were enrolled from April 2013 to December 2018. Only newly diagnosed patients with no history of medication use were included in the study. Patients with heart disease, hypertension, and diabetes were excluded, per physical exam and laboratory tests. A total of 91 subjects initially were selected. Exclusion criteria included incomplete postoperative echocardiography (19 patients did not strictly review cardiac ultrasound, according to follow-up time) and sound window difference (this excluded an additional 12 patients). Finally, 60 patients were identified as the final EA group subjects. A normal control group was comprised of 30 healthy subjects from the clinic.

Methods: Transthoracic echocardiography via commercially available GE company's Vivid E9 color Doppler ultrasound machine was performed by a fixed experienced ultrasound physician for patients with Ebstein anomaly and standard two-dimensional (2D) color Doppler images were collected, according to ASE [Lang 2005]. The probe frequency was set between 3.2-6.4 MHz.

The patients were examined in the left decubitus position with EKG connected in a relaxed mood. To minimize errors, all echocardiographic measurements in three consecutive cardiac cycles were required. Images were stored in the hard disk and imported into the EchoPAC workstation. All echocardiographic parameters were calculated and analyzed offline.

General parameters and tissue Doppler measurement: Routine measurement of right ventricular end-diastolic diameter (RVEDD), right ventricular end-systolic diameter (RVESD), right ventricular end-diastolic area (RVEDA), and right ventricular end-systolic area (RVESA) in the RV free wall and interventricular septum tissue in tissue Doppler imaging (TDI) were performed. High time resolution (frame rate $>160 \mathrm{~Hz}$ ) color TDI was collected, each image containing 


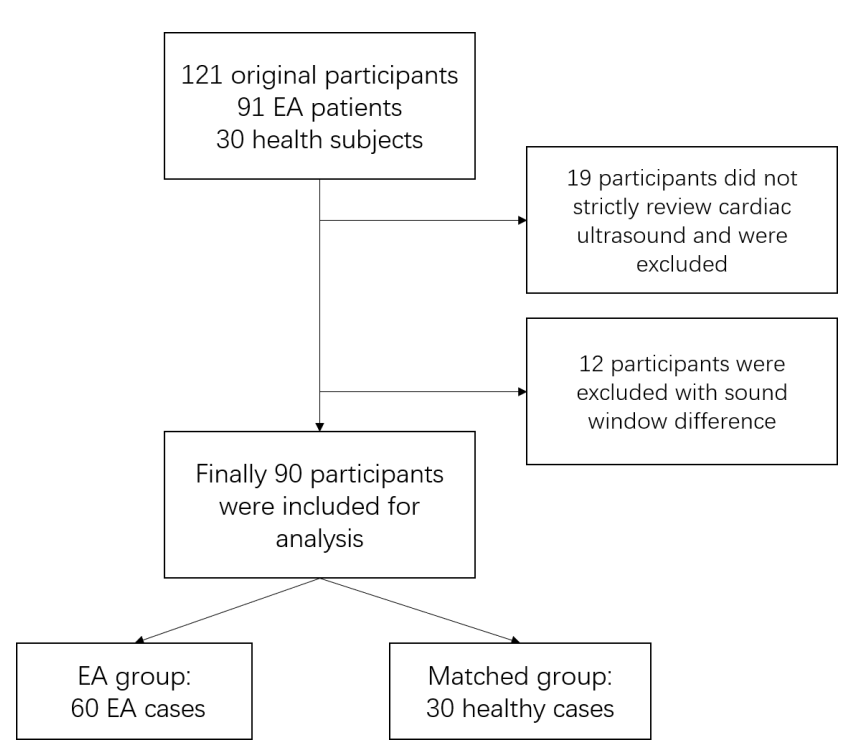

Figure 1. Detailed flow chart of included participants.

three complete cardiac cycles. Six parts (the midpoint of the upper, middle and lower part of the right ventricular free wall and the interventricular septum myocardium, which are the apex, middle and base of right ventricular free wall and interventricular septum myocardium). The data were generated by offline analysis with professional post-processing software (EchoPAC®, QLAB9.1). For strain imaging analysis, an offset of $12 \mathrm{~mm}$ was adopted (Figure 2). Strain was expressed in percentage, and the unit of the strain rate is s-1 (Figure 3). Doppler measurements over three consecutive cardiac cycles with normal breathing were evaluated. Negative strain values indicate tissue shortening, and smaller values (higher absolute values) represent better right ventricular systolic function.

Statistical analysis: The sample size was calculated using the expected correlation coefficient, power of the study (80\%) and significance level (95\%). The measurement data were based on skewness and kurtosis $(P>.05)$. We used mean and student t-test. Continuous variables were presented as mean \pm standard deviation (SD). Differences in follow-up data between the normal control group and EA group were measured with independent samples t-test analysis. The follow-up data of the EA group and control group were analyzed by one-way ANOVA. $P<.05$ was considered to be of significant statistical difference. All statistical analyses were performed with SPSS version 20 (SPSS Inc., Chicago, IL, USA).

\section{RESULTS}

Clinical and echocardiographic characteristics of the EA patient and control groups: Sixty patients with EA were included in the study (35 females and 25 males), with an average age of $17.43 \pm 15.18$ years. Detailed flow charts are shown in Figure 1. There were 30 subjects in the control group (13 females and 17 males), with an average age of $23.53 \pm 15.07$ years. There was no significant difference between the EA

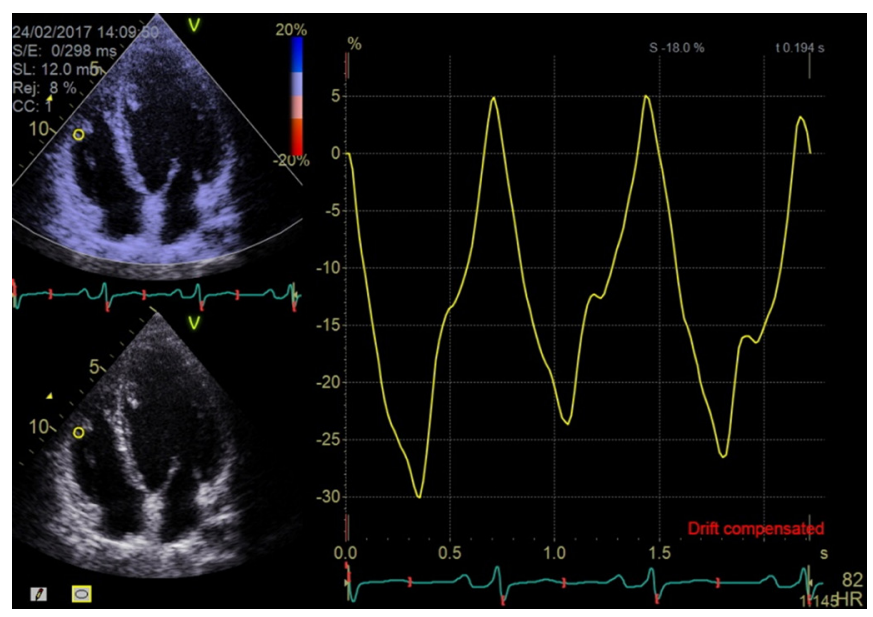

Figure 2. Measurement of RV strain in the mid free wall segment.

patients and control group regarding age $(P=.694)$ and sex $(P=.189)$ (Table). RVEDD, RVESD, RVEDA, and RVESA values in the EA group were significantly higher than those in the control group $(P=.000)$.

Comparison of follow-up data of EA group at various stages during perioperative period: As shown in Tables 2, 3, and 4 in this study, we used transthoracic echocardiography to obtain data of RVEDD, RVESD, RVEDA, RVESA RV strain and strain rate to evaluate changes in RV function before and after Ebstein anomaly correction, and to understand the implication of different methods for assessing right ventricular function after EA.

1) The RVEDD of the EA group: The values measured at 1 week and 3 months after surgery were significantly lower than preoperative values $(P=.000)$. Postoperative 3 -month RVEDD was higher than that of the control group $(P=.129)$.

2) The RVESD of the EA group: The values at 1 week and 3 months after surgery were significantly lower than those before surgery $(P=.000$ and $P=.002$, respectively).

3) The RVEDA of the EA group: The values measured before surgery were higher than those in the control group $(P$ $=.000)$. Post-op 1-week and 3 months values were lower than pre-op values $(P=.000)$.

4) The RVESA of the EA group: The RVESA at 1 week and 3 months after surgery were reduced $(P=.741)$. Postop 3-month value was higher than that of the control group $(P=.001)$.

Comparison of right ventricular strain and strain rate during perioperative period in the EA group: In this study, the basal segmental strain of ventricular septum in the EA group was noted to increase at 1 week and 3 months after surgery, and it significantly increased at 3 months after surgery $(P=.000)$. Strain values of the middle ventricular septum and apical segment in the EA group increased at 1 week $(P=$ .727 and $P=.072$, respectively) and 3 months after operation $(P=.201$ and $P=.08$, respectively). Basal and mid-segment strain of the free wall of the right ventricle in the EA group rose one week after surgery compared with preoperative values $(P=.139$ and $P=.058$, respectively). A significant increase was 


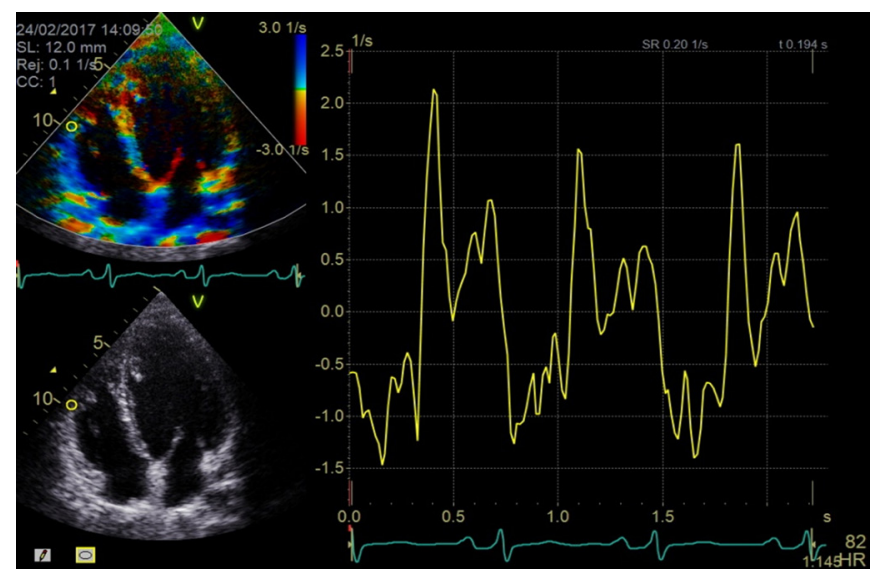

Figure 3. Measurement of RV strain rate in the mid free wall segment.

found 3 months after the surgery compared with the preoperative measurement $(P=.000)$. In the EA group, the apical strain of the right ventricular free wall was decreased at 1 week and 3 months after the operation $(P=.084$ and $P=.197$, respectively), as shown in Table 3.

Ventricular septal strain rate: The basal segment strain rate of ventricular septum measured in the EA at 1 week and 3 months after surgery significantly increased compared with the preoperative SR $(P=.002$ and $P=.000$, respectively). The strain rate $(\mathrm{SR})$ of the mid-segment of ventricular septum in the EA group showed that the SR at 1 week and 3 months after surgery increased compared with that before surgery ( $P=.411$ and $P=.055$, respectively). The strain rate of apical segment in interventricular septum in the EA group showed that the SR at 1 week and 3 months after surgery were lower than that before surgery $(P=.803$ and $P=.821$, respectively).

Right ventricular free wall strain rate: In this study, we measured the free wall basal segment strain rate in the EA group. The results showed that the SR at 1 week was increased $(P=.075)$. SR at 3 months after surgery also was higher than before surgery $(P=.026)$. The SR of the RV free wall middle segment in the EA group at 3 months after surgery was significantly higher than that before surgery $(P=.000)$. Apical free wall RV strain rate at 1 week and 3 months after surgery were lower than that before surgery $(P=.812$ and $P=.221$, respectively), as listed in Table 4.

\section{DISCUSSION}

Myocardial strain imaging (S) and strain rate (SR) are TDI-derived models. Strain can be obtained by the magnitude of the deformation between contraction and end-diastole used as a reference point. SR measures the deformation rate of the tissue segment, and it is expressed as S-1. Strain and strain rate imaging can be used to distinguish between active and passive movements of the myocardial segment and can be used to evaluate local myocardial function, such as longitudinal shortening of the myocardium, and comprehensive myocardial function. The above two parameters can be
Characteristics of baseline data of EA group and control group

\begin{tabular}{lccc}
\hline & EA group & Control group & $P$ \\
\hline Number of cases & 60 & 30 & \\
Gender (male/female) & $25 / 35$ & $17 / 13$ & .189 \\
Age & $17.43 \pm 15.18$ & $23.53 \pm 15.07$ & .694 \\
$\operatorname{RVEDD}(\mathrm{cm})$ & $4.39 \pm 0.99$ & $3.29 \pm 0.21$ & .000 \\
$\operatorname{RVESD}(\mathrm{cm})$ & $3.54 \pm 0.96$ & $2.69 \pm 0.29$ & .000 \\
$\operatorname{RVEDA}\left(\mathrm{cm}^{2}\right)$ & $21.79 \pm 6.85$ & $14.25 \pm 2.29$ & .000 \\
$\operatorname{RVESA}\left(\mathrm{cm}^{2}\right)$ & $14.53 \pm 6.21$ & $8.86 \pm 2.13$ & .000 \\
\hline
\end{tabular}

RVEDD: right ventricular end-diastolic diameter, RVESD: right ventricular end-systolic diameter, RVEDA: right ventricular end-diastolic area, RVESA: right ventricular end-systolic area

obtained using TDI or speckle tracking 2DE (2DSTE) technology. The correlation between TDI and 2DSTE-derived RV longitudinal strain values appears to be moderate.

However, the two techniques are considered sufficiently accurate to distinguish between physiological and pathological conditions [Teske 2008]. The formula for calculating strain is $=(\mathrm{L}-\mathrm{Lo}) / \mathrm{Lo} . \mathrm{L}$ is the immediate length, and Lo is the initial length. Myocardial prolongation or thinning strain is positive, while myocardial shortening or thickening strain is negative. This study evaluated right ventricular function by peak systolic strain analyzed by tissue Doppler imaging.

Bos et al found in the study of RV strain that the heart strain in patients with corrected aortic transposition (CTGA) was significantly lower than that in a normal control group, suggesting that CTGA patients have a decline in right heart pump function [Bos 2006]. Kjaergaard et al reported changes in RV strain in patients with pulmonary embolism, during the acute phase and recovery stage [Kjaergaard 2006]. Although the number of patients is small, this was the first report of abnormal movement of RV free wall by strain analysis.

The longitudinal strain value of RV is closely related to the prognosis of pulmonary hypertension. It was reported that the longitudinal strain of the right ventricle was a predictor of cardiovascular events [Rasalingam 2011]. RV function is highly dependent on load, which affects myocardial contractibility. Therefore, it has been shown that in patients with RV pressure overload, RV strain is significantly lower than that in normal subjects with or without myocardial contractility impairment [Prakasa 2007]. The clinical and prognostic value of RV strain also has been proven in not only patients with pulmonary hypertension [Hardegree 2013], but also patients with congenital heart disease [Lu 2013], myocardial infarction [Antoni 2010], cardiomyopathy with high risk of malignant ventricular arrhythmias [Aneq 2012], heart failure, and patients with LV assistive devices [Hayek 2014].

The results of this study showed that $\mathrm{S}$ and $\mathrm{SR}$ in the Ebstein anomaly patient group were significantly reduced compared with those in the control group, implying that myocardial systolic function was impaired before surgery. 
The results obtained in this study showed that the right ventricular S and SR of EA patients were elevated after surgery than before. It is most prominent in the basal segment, middle segment of RV free wall, and basal segment of the interventricular septum.

The right heart anatomy and hemodynamic characteristics are as follows: The right atrial wall of the heart is thin. The right ventricle is a high-capacity low-pressure pump. There are three leaflets attached to the periphery of the atrioventricular junction. The anterior leaflet, posterior leaflet, and septal leaflet together are called the right atrioventricular valve (also known as the tricuspid valve). The three leaflets vary in size with the anterior leaflet dominating. The tricuspid annulus is an elliptical saddle-shaped structure that becomes more rounded when the right ventricle expands. The integrity of the tricuspid device is closely associated with the size and function of the right ventricle. Volume load of the right ventricle (such as tricuspid regurgitation or a large atrial septal defect) or right ventricular pressure overload (such as pulmonary valve stenosis or pulmonary artery hypertension), or both, can impair tricuspid valve function. Chronic volume overload causes right ventricle dilation. Chronic pressure overload causes right ventricular hypertrophy to preserve right ventricular ejection function. The right ventricle is quite sensitive to acute pressure overload, such as a massive pulmonary embolism, in which acute right heart failure can be seen when the average pulmonary artery pressure exceeds 30-35 mmHg. Tricuspid regurgitation can cause RV failure, manifested as hepatomegaly, ascites, edema, and low cardiac output syndrome.

In patients with RV dysfunction, the aforementioned characteristics of the right ventricle is remarkably augmented, and standard echocardiographic parameters often fail to accurately delineate the local myocardium. In a concurrent echocardiographic study [Cameli 2012], we have demonstrated a good negative correlation between longitudinal strain of the right ventricle and the RV Tei index. The right ventricular free wall strain is most helpful in the diagnosis accuracy. This is probably because that strain of right ventricular free wall more reliably reflects right heart function than the strain of right ventricular longitudinal strain. In fact, the interventricular septum is shared by two ventricles and its strain pattern also is affected by LV systolic function. In the classical anatomy pattern of EA, the posterior leaflet and septal leaflet move downward; a large part of interventricular septum serves as the atrialized right ventricle. In our study, strain and strain rate of the basal segment of the interventricular septum went up at 3 months after surgery $(P<.01)$. While strain and strain rate were also noted at 1 week and 3 months after operation in the middle and apical segments of interventricular septum $(P>.05)$, the difference was not statistically significant. On the other hand, basal and mid-segment strain and strain rate of the free wall of the right ventricle in the EA group rose 1 week after surgery compared with preoperative values $(P>.05)$. A significant increase was found 3 months after the surgery compared with the preoperative measurement $(P<.01)$. The conclusion is that we can monitor the right ventricular systolic function recovery by evaluating the strain and strain rate of the right ventricular free wall base and middle segment as well as the base of the interventricular septum at 3 months after operation.

However, there are several limitations in this study. First, our study is lacking in reference of MRI evaluation of RV volume and systolic function in order to make a fair comparison. Second, TDI is angle-dependent, bears prominent systolic deviation, and requires a high frame rate of strain curve. Aside from the technical obstacles, patient age and heart rate potentially can distort the accuracy and precision of TDI measurement of strain and strain rate. Third, TDI measurement is mainly limited to the apical four-chamber view. Therefore, only RV longitudinal strain is evaluated. But longitudinal shortening contributes more to $\mathrm{RV}$ contraction than circumferential shortening [Kukulski 2000]. Finally, reading data is time consuming and requires offline analysis. Even though these variables are very helpful in understanding cardiac physiology, their feasibility in clinical practice remains to be proven.

In conclusion, the measurement of RV strain and strain rate on tissue Doppler imaging can be employed to assess the preoperative and postoperative RV function, prove the positive effect of tricuspid valve repair on right heart function, and offer more insight on right heart function evaluation.

\section{REFERENCES}

Aneq MA, Engvall J, Brudin L, Nylander E. 2012. Evaluation of right and left ventricular function using speckle tracking echocardiography in patients with arrhythmogenic right ventricular cardiomyopathy and their first degree relatives. Cardiovasc Ultrasound. 10:37-41.

Antoni ML, Scherptong RW, Atary JZ, et al. 2010. Prognostic value of right ventricular function in patients after acute myocardial infarction treated with primary percutaneous coronary intervention. Circ Cardiovasc Imaging. 3:264-271.

Bos JM, Hagler DJ, Silvilairat S, et al. 2006. Right Ventricular Function in Asymptomatic Individuals with a Systemic Right Ventricle. J Am Soc Echocardiogr. 19:1033-1037.

Cameli M, Lisi M, Righini FM, et al. 2012. Right ventricular longitudinal strain correlates well with right ventricular stroke work index in patients with advanced heart failure referred for heart transplantation. J Card Fail. 18:208-215.

Hardegree EL, Sachdev A, Villarraga HR, et al. 2013. Role of serial quantitative assessment of right ventricular function by strain in pulmonary arterial hypertension. Am J Cardiol. 111:143-148.

Hayek S, Sims DB, Markham DW, Butler J, Kalogeropoulos AP. 2014. Assessment of right ventricular function in left ventricular assist device candidates. Circ Cardiovasc Imaging. 7:379-389.

Kjaergaard J, Petersen CL, Kjaer A, Schaadt BK, Oh JK, Hassager C. 2006. Evaluation of right ventricular volume and function by $2 \mathrm{D}$ and 3D echocardiography compared to MRI. Eur J Echocardiography. 7:430-438.

Kukulski T, Hubbert L, Arnold M, et al. 2000. Normal regional right ventricular function and its change with age: a Doppler myocardial imaging study. J Am Soc Echocardiography. 13:194-204.

Lang RM, Bierig M, Devereux RB, et al. 2005. Recommendations for 
chamber quantification: a report from the American Society of Echocardiography's Guidelines and Standards Committee and the Chamber Quantification Writing Group, developed in conjunction with the European Association of Echocardiography, a branch of the European Society of Cardiology. J Am Soc Echocardiogr. 18:1440-1463.

Lu JC, Ghadimi Mahani M, Agarwal PP, Cotts TB, Dorfman AL. 2013. Usefulness of right ventricular free wall strain to predict quality of life in "repaired" tetralogy of Fallot. Am J Cardiol. 111:1644-1649.

Prakasa KR, Wang J, Tandri H, et al. 2007. Utility of tissue Doppler and strain echocardiography in arrhythmogenic right ventricular dysplasia cardiomyopathy. Am J Cardiol. 100:507-512.
Rasalingam R, Johnson SN, Bilhorn KR, et al. 2011. Transthoracic echocardiographic assessment of continuous-flow left ventricular assist devices. J Am Soc Echocardiography. 24:135-148.

Teske AJ, De Boeck BW, Olimulder M, Prakken NH, Doevendans PA, Cramer MJ. 2008. Echocardiographic assessment of regional right ventricular function: a head-to-head comparison between 2-dimensional and tissue Doppler-derived strain analysis. J Am Soc Echocardiography. 21:275-283.

Zhang X, Wu Q, Dong B, Li H, Zhang M, Jin Y. 2018. The outcomes of operation for 237 patients with Ebstein anomaly. Chin J Surg. 56: 418-421. 